\title{
Prevalence of third molar impaction in patient with mandibular anterior teeth crowding
}

\author{
Tan Chun Wei*, Eky Soeria Soemantri*, Iwa Rahmat Sunaryo* \\ Department of Orthodontics, Faculty of Dentistry, Universitas Padjadjaran, Indonesia
}

\begin{abstract}
Introduction: Third molar impaction has been a controversial topic among clinician when it comes to mandibular anterior teeth crowding. The aim is to know the prevalence of third molar impaction in patient with mandibular anterior teeth crowding in Orthodontic Department, Dental Hospital Universitas Padjadjaran (RSGM UNPAD), Indonesia. Methods: This was a descriptive research, with non-probability sampling obtained from a population with full arch 32 teeth. Totaling 54 samples from year 2011 to 2014 were obtained. The age of sample range from 15 to 25 years old. Also all panoramic radiographs taken from the year 2011 to 2014 were examined using a computer.The position of third molars were determined by Winter's classification using angle formed between the intersected longitudinal axes of the second and third molars and anterior teeth status by using Little's irregularities index. Results: This study obtained data that as much as $68.52 \%$ mesioangular third molar, $19.44 \%$ distoangular, $4.63 \%$ horizontal, $1.85 \%$ buccolingual, and $0 \%$ others. In mesioangular impaction condition there was crowding $83.78 \%$, in distoangular condition there was $100 \%$ crowding, in horizontal position crowding found $100 \%$, while at buccolingual crowding found $100 \%$. Conclusion: In mesioangular impaction condition there was crowding $83.78 \%$, in distoangular condition there was $100 \%$ crowding, in horizontal position crowding found $100 \%$, while at buccolingual crowding found $100 \%$. In all positions the third molar impaction was accompanied by crowding of the mandible anterior teeth, with the largest percentage of impaction was the mesioangular position.
\end{abstract}

Keywords: prevalence; impacted third molar; anterior teeth crowding; panoramic radiograph

\section{INTRODUCTION}

It is a pathological condition that the impacted tooth cannot or will not erupt into its normal functioning position. Also, it have shown by some research works that impacted third molar weakens the angle of mandible and susceptible to fracture and is implicated in the etiology of lower arch crowding, Temporomandibular joint (TMJ) disorders, vague orofacial pain and neuralgias. ${ }^{1}$
In modern populations, the prevalence of third molar impaction is higher than other teeth and mandibular third molar is by far the most frequently impacted tooth after the maxillary third molar. ${ }^{2-4}$ The reason for this is probably they are the last teeth erupting into the dental arch therefore the chance of space deficiency for their eruption is high. ${ }^{5}$ In addition, third molar varies more than the other molars in terms of shape, size, timing of eruption, and even tendency toward 
impaction. ${ }^{6}$ Studies found that females are earlier in the root development of other permanent teeth, while males are earlier in the case of third molar. ${ }^{7}$ A study stated that the frequency of third molar impaction was quite high with $84 \%$ in age group of 15-25 years, this maybe due to coincidence of this age with third molar eruption and initial complaints are usually encountered during eruption phases. ${ }^{8}$

A case report from Libyan population, the distribution of impacted third molars in angulation classification showed that mesioangular impaction was the most frequent (34.6\%) followed by vertical (31.3\%) and distoangular (27.7\%). Besides, there were significantly more mesioangular impactions in the mandible $(78.5 \%)$ and more distoangular (66.9\%) and vertical $(56.4 \%)$ impactions in the maxilla. ${ }^{9}$ Mesioangular impactions are probably the most common type and this may be due to their late development and maturation, path of eruption and lack of space in mandible at later age. ${ }^{1}$

A study result from Sidlauskas ${ }^{10}$ indicated that general lower dental arch crowding is an essential feature of the completed permanent dentition with $90 \%$ cases of space lacking. However, some tendency for crowding in the anterior part of lower dental arch was more expressed in the group with third molars, than with agenesis. ${ }^{10}$

Based on the problems of impaction and crowding that are commonly found, the purpose of this study was to obtain prevalence data in patients with molar third impaction and crowding mandibular anterior teeth. The data can be used as a study material for prevention of occurrence of impaction and crowding, as well as become a consideration in crowding and malocclusion manajement accompanied by third molar impaction.

\section{METHODS}

This was a descriptive research, with nonprobability sampling obtained from a population with full arch 32 teeth. Totaling 54 samples from year 2011 to 2014 were obtained include models and panoramic radiograph. The age of sample range from 15 to 25 years old. The position of third molars were determined by Winter's classification using angle formed between the intersected longitudinal axes of the second and third molars and anterior teeth status by using Little's irregularities index. Datas were collected in the form of secondary data from Orthodontic Department of Rumah Sakit Gigi dan Mulut (RSGM), Universitas Padjadjaran, Indonesia. All panoramic radiographs taken from the year 2011 to 2014 were examined using a computer. The sampling method used is the non probability sampling technique, based on the selection criteria. The sample for this research include age between 15 to 25 years old, patients with 32 full arch teeth and present with clear anatomical landmark and good exposure. Exclusive criteria include those who with systemic disease that alter the lower jaw growth development.

The impacted third molars were recorded based on Winter's classification. According to the position of impacted third molars to the long axis of the second molar, classification was done as mesioangular, horizontal, vertical, distoangular, and those rare angulations are classified in others. Measurement of angulations of third molars were

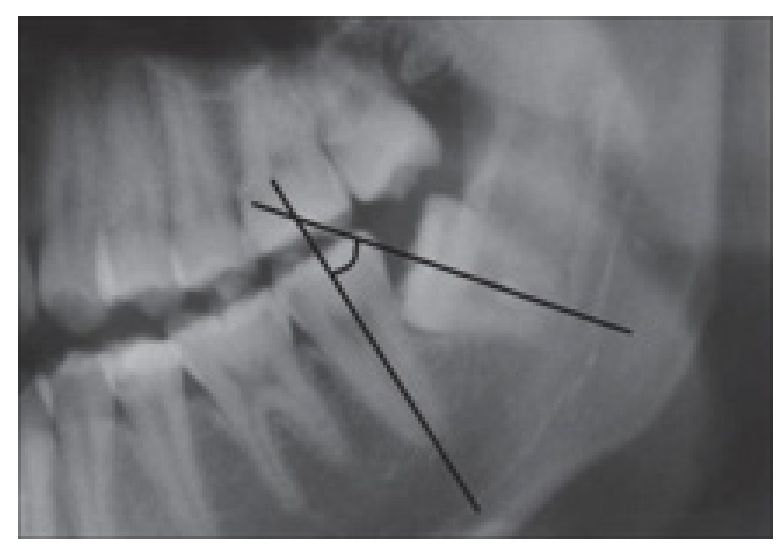

Figure 1. Measurement of angulation of third molar on panoramic radiograph to determine the classification of impacted teeth. ${ }^{11}$

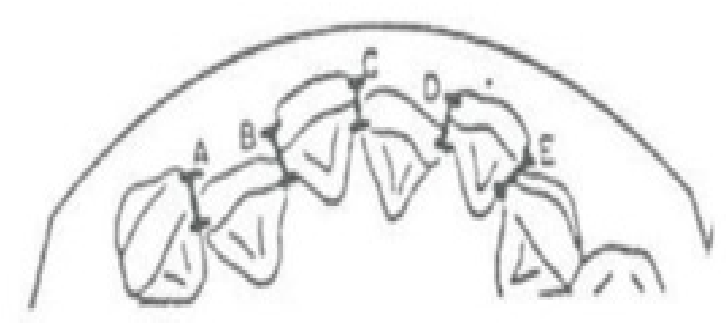

Figure 2. Measurement of anatomic contact points of each mandibular incisors to determine mandibular anterior teeth crowding. ${ }^{12}$ 
determined by tracing panoramic radiographs using digital protractor by the angle formed between intersected longitudinal axes of the second molar and the third molar (Fig.1).

The examination of mandibular anterior teeth crowding was done by using the Little's irregular index, a scoring method that involve measurement of 5 liner displacement (labiolingually) of anatomic contact points of each mandibular incisor from the adjacent tooth. Five displacements from the mesial aspect of the right canine to the mesial aspect of left canine were examined.

The measurements were obtained directly from the mandibular cast. Caliper was held parallel to the occlusal plane (Fig. 2). Each of the five measurements represents a horizontal linear distance between the anatomic points of the adjacent teeth. Scoring were given for each patient cast according to Little's irregularity index, 0 for perfect alignment, 1-3 for minimal, 4-6 moderate, 7-9 severe and 10 for very severe. So index more than 0 was considered crowding.

\section{RESULT}

Table 1 shows the percentage of male and female samples that were included for this research. Based on Table 1. $68.52 \%$ of the sample were female whereas $31.48 \%$ of the sample were male. The ratio for gender is around 2:1 female: male.

Table 1. Sample mean, persentage and standard deviation according to gender.

\begin{tabular}{ccccc}
\hline Gender & Mean & $\mathrm{N}$ & Percentage & Sd \\
\hline Female & 20.54 & 37 & $68.52 \%$ & 2.4449 \\
Male & 19.06 & 17 & $31.48 \%$ & 2.6192 \\
\hline Total & 20.70 & 54 & $100 \%$ & 2.6916 \\
\hline
\end{tabular}

Table 2. Frequency of impaction status in patients.

\begin{tabular}{ccc}
\hline Impaction Status & Frequency & Percent (\%) \\
\hline Mesioangular & 74 & 68.52 \\
Distoangular & 6 & 5.56 \\
Vertical & 21 & 19.44 \\
Horizontal & 5 & 4.63 \\
Others & 0 & 0 \\
Buccolingual & 2 & 1.85 \\
\hline Total & 108 & 100 \\
\hline
\end{tabular}

Within all the patients, mesioangular occupy $68.52 \%, 19.44 \%$ for vertical, $5.56 \%$ distoangular, $4.63 \%$ horizontal, $1.85 \%$ for buccolingual and $0 \%$ for others, as seen at table 2 below.

Table 3 shows the frequency of anterior teeth status in patients in orthodontic department of RSGM Unpad, Indonesia. Of all the patients, crowding occupy the most with $87.03 \%, 7.41 \%$ for normal, $5.56 \%$ for anterior crossbite and $0 \%$ for spacing. Male has higher anterior crossbite than female with $11.76 \%$ over $2.70 \%$. Female also has higher normal status than male with $8.11 \%$ over $5.88 \%$.

Table 4 shows distribution of third molar impaction in Orthodontic Department of RSGM Unpad, Bandung, with mandibular anterior teeth crowding Within all the anterior teeth status, crowding occupy the most in all type of third molar impaction. The mesioangular impaction patient who also has anterior teeth crowding were $83.78 \%$, vertical impaction with anterior crowding occupy $90.48 \%$, distoangular has $100 \%$ crowding, horizontal occupy $100 \%$ crowding and buccolingual $100 \%$ crowding.

Table 3. Frequency of anterior teeth status in patients in Orthodontic Department of RSGM.

\begin{tabular}{lll}
\hline \multicolumn{1}{c}{ Anterior teeth status } & Frequency & Percent \\
\hline Crowding & 47 & $87.03 \%$ \\
Normal & 4 & $7.41 \%$ \\
Anterior Crossbite & 3 & $5.56 \%$ \\
Spacing & 0 & $0 \%$ \\
\hline Total & 54 & $100 \%$ \\
\hline
\end{tabular}

Table 4. Distribution of third molar impaction with mandibular anterior teeth crowding in patients in Orthodontic Department of RSGM.

\begin{tabular}{ccc}
\hline & Frequency & Crowding \\
\hline $\begin{array}{c}\text { Vertical } \\
\left(10^{\circ} \text { to }-10^{\circ}\right)\end{array}$ & 21 & $19(90.48 \%)$ \\
$\begin{array}{c}\text { Mesioangular } \\
\left(11^{\circ} \text { to } 79^{\circ}\right)\end{array}$ & 74 & $62(83.78 \%)$ \\
$\begin{array}{c}\text { Horizontal } \\
\left(80^{\circ} \text { to } 100^{\circ}\right)\end{array}$ & 5 & $5(100 \%)$ \\
$\begin{array}{c}\text { Distoangular } \\
\left(-11^{\circ} \text { to }-79^{\circ}\right)\end{array}$ & 6 & $6(100 \%)$ \\
$\begin{array}{c}\text { Others } \\
\left(110^{\circ} \text { to }-80^{\circ}\right)\end{array}$ & 0 & $0(0 \%)$ \\
Buccolingual & 2 & $2(100 \%)$ \\
\hline
\end{tabular}


The distribution of third molar impaction position, were seen in Table 4. In vertical impaction there were $90.48 \%$ of them experiencing crowding, while in mesioangular position there was $83.78 \%$. Horizontal, distoangular, buccolingual and other impaction showed $100 \%$ with crowding on mandibular anterior teeth.

\section{DISCUSSION}

The insidence of third molar impaction is higher than other teeth and mandibular third molar is by far the most frequently impacted tooth after the maxillary third molar, their account for 98 per cent of all impacted teeth. ${ }^{2-4}$

Several mechanisms have been suggested to explain the aetiology of third molar impaction, these include: impaction of third molar occurs as a result of retardation of facial growth, shortage of space in the third molar region, vertical direction of the condylar growth associated with low resorption of the anterior border of the ramus, the distal direction of the eruption of the other teeth, low mandibular growth rate resulting in a reduction in the length of the jaws, early physical maturity, and late third molar mineralization. But the main reason for this is probably they are the last teeth erupting into the dental arch therefore the chance of space deficiency for their eruption is high. ${ }^{5}$

According to the analysis in this study, both male and female, mesioangular occupy the most impaction based on Winter's classification. The reason for this may be due to their late development and maturation, path of eruption and lack of space in mandible at later age. ${ }^{1}$ Beside mandibular growth, tooth bud angulation also take part, typically tooth bud is mesially angulated and with the variation growth, some third molar might experience increased mesial angulation during early and late adolescence. ${ }^{16}$

These data has the same pattern to some research study in which $62.9 \%$ of mesioangular impaction studied by Kruger et al. $60 \%$ by Quek et al. and $50 \%$ by Hattab et al. and the second most impacted classification is vertical which occupy $19.44 \%$ which same pattern with Hattab et al. $34 \%$ and Kruger et al. $11.9 \%$ but different with Quek et al. being 9.5\%, less than horizontal impaction. Distoangular and horizontal impaction hold almost same amount which are $5.56 \%$ and $4.63 \%$ in this study, same pattern with Hattab et al. with distoangular $5 \%$ and horizontal $5 \%$, a bit less on Kruger et al. with distoangular $1.4 \%$ and horizontal $1 \%$ and a bit higher on Quek et al. with distoangular $9.8 \%$ and horizontal $17.6 \% .17-19$

In this study, the frequency of female in number of impaction is higher than male, the reason for this maybe due to the consequence of difference between the growth of males and females. Females usually stop growing when the third molars just begin to erupt, whereas in males, the growth of the jaws continues during the time of eruption of the third molars, creating more space for third molar eruption. ${ }^{20}$

In the reference of Winter's classification and based on the order from most to the least in this study, mesioangular is the most followed by vertical, distoangular, horizontal, buccolingual and others but in male left mandibular being the only one exception with horizontal more than distoangular. The reason being the exception might be the little samples in the horizontal and distoangular compared to the main impaction such as mesioangular and vertical as there is no data on the male right mandibular horizontal and male left mandibular distoangular. There is no data obtained on others category in both male and female as well and there is only two buccolingual impaction measured, both are in the same female on both sides and none for male.

A study indicated that general lower dental arch crowding is an essential feature of the completed permanent dentition with $90 \%$ cases of space lacking. ${ }^{10}$ Based on this study, crowding occupy the most anterior teeth condition pattern both in female $(89.19 \%)$ and male $(82.35 \%)$, with total $87.03 \%$ occupy the whole population. In normal condition, female occupy $8.11 \%$ and male slightly lower, $5.88 \%$, and $7.41 \%$ in total. In anterior crossbite, female hold $2.70 \%$ and male $11.76 \%$ slightly higher, and $5.56 \%$ total calculation in both female and male. There is no spacing data measured in this study. It is apparent that if dental arch dimensions are reduced, dental crowding must increase. Factors responsible for dental arch reduction may vary from one person to another, and many factors, acting together or at different stages of development, may contribute to lower dental arch crowding. 


\section{CONCLUSION}

In mesioangular impaction condition there was crowding $83.78 \%$, in distoangular condition there was $100 \%$ crowding, in horizontal position crowding found $100 \%$, while at buccolingual crowding found $100 \%$. In all positions the third molar impaction was accompanied by crowding of the mandible anterior teeth, with the largest percentage of impaction was the mesioangular position.

\section{REFERENCES}

1. Hashemipour MA, Tahmasbi-Arashlow M, Fahimi-Hanzaei F. Incidence of impacted mandibular and maxillary third molars: A radiographic study in a southeast iran population. Med Oral Patol Oral Cir Bucal 2013;18(1):1-6.

2. Bishara SE, Andreasen G. Third molars: A review. Am J Orthod 1983;83(2):131-7.

3. Grover PS, Lorton L. The incidence of unerupted permanent teeth and related clinical cases. Oral Surg Oral Med Oral Pathol 1985 Apr;59(4):420-5.

4. Alling III CC, Catone GA. Management of impacted teeth. J Oral Maxillofac Surg 1993 Jan;51 (1 suppl1):3-6.

5. Breik 0, Grubor $D$. The incidence of mandibular third molar impactions in different skeletal face types. Aust Dent J 2008;53(4):320-4.

6. Abu Alhaija ESJ, Albhairan HM, Alkhateeb SN. Mandibular third molar space in different antero-posterior skeletal patterns. Eur J Orthod 2011;33(5):570-6.

7. Gunst K, Mesotten K, Carbonez A, Willems G. Third molar root development inrelation to chronological age: A large sample sized retrospective study. Forensic Sci Int 2003;136(1-3):52-7.

8. Amanat N, Rcs FDS, Mirza D, Rizvi KF, Rcs D. Pattern of third molar impaction: frequency and types among patients attending urban teaching hospital of Karachi. Pakistan Oral Dent J 2014;34(1):1-4.
9. Hatem M, Bugaighis I, Taher EM. Pattern of third molar impaction in Libyan population: $\mathrm{A}$ retrospective radiographic study. Saudi J Dent Res 2016;7(1):7-12.

10. Sidlauskas A, Trakiniene G. Effect of the lower third molars on the lower dental arch crowding. Stomatol/issued by public Inst Odontologijos Stud 2006;8(3):80-4.

11. Kanneppady SK, Balamanikandasrinivasan, Kumaresan R, Sakri SB. A comparative study on radiographic analysis of impacted third molars among three ethnic groups of patients attending AIMST Dental Institute, Malaysia. Dent Res J (Isfahan) 2013 May;10(3):353-8.

12. Madhusudhan V. Prevalance of mandibular anterior crowding in Tumkur population. J Dent Sci Res 2011;2(2):6-8.

13. Björk A, Jensen E, Palling M. Mandibular growth and third molar impaction. Acta Odontol Scand 1956;14:231-72.

14. Richardson ME. The etiology and prediction of mandibular third molar impaction. Angle Orthod 1977 Jul;47(3):165-72.

15. Altonen M, Haavikko K, Mattila K. Developmental position of lower third molar in relation to gonial angle and lower second molar. Angle Orthod 19770ct;47(4):249-55.

16. Richardson ME. Development of the lower third molar from 10 to 15 years. Angle Orthod 1973Apr;43(2):191-3.

17. Kruger $\mathrm{E}$, Thomson $\mathrm{M}$. Third molar outcomes from age 18 to 26: Findings from a populationbased New Zealand longitudinal study. Oral Surg Oral Med Oral Pathol Oral Radio Endod 2001;92:150-5.

18. Quek SL, Tay CK, Tay KH, Toh SL, Lim KC. Pattern of third molar impaction in a Singapore Chinese population: a retrospective radiographic survey. Int J Oral Maxillofac Surg 2003;32(5):548-52.

19. Hattab FN, Rawashdeh A. Impaction status of third molars in Jordanian students. oral Surg Oral Med Oral Pathol Endod 1995;79:24-9.

20. Bishara SE. Impacted maxillary canines: a review. Am J Orthod Dentofacial Orthop 1992 Feb;101(2):159-71. 